\title{
Visual Perception of Mean Relative Phase and Phase Variability
}

\author{
Frank T. J. M. Zaal and Geoffrey P. Bingham \\ Indiana University Bloomington
}

\author{
Richard C. Schmidt \\ College of the Holy Cross
}

\begin{abstract}
Perception of relative phase and phase variability may play a fundamental role in interlimb coordination.
- This study was designed to investigate the perception of relative phase and of phase variability and the stability of perception in each case. Observers judged the relative phasing of two circles rhythmically moving on a computer display. The circles moved from side to side, simulating movement in the frontoparallel plane, or increased and decreased in size, simulating movement in depth. Under each viewing condition, participants observed the same displays but were to judge either mean relative phase or phase variability. Phase variability interfered with the mean-relative-phase judgments, in particular when the mean relative phase was $0^{\circ}$. Judgments of phase variability varied as a function of mean relative phase. Furthermore, the stability of the judgments followed an asymmetric inverted U-shaped relation with mean relative phase, as predicted by the Haken-Kelso-Bunz model.
\end{abstract}

In the early eighties, Kugler, Turvey, and Kelso, among others, introduced the concepts of nonlinear dynamics into the study of human movement, thereby building on Bernstein's (1967) important insight that perception-action systems should be regarded as coordinative structures that are task specific and soft molded (Kelso, Holt, Rubin, \& Kugler, 1981; Kugler, Kelso, \& Turvey, 1980; Kugler \& Turvey, 1987). Taking rhythmicity as paradigmatic of human movement, they noted that cyclical movements are sustained in spite of the universal tendency for order to diminish and cease as described by the second law of thermodynamics (cf. Yates, 1982). They suggested therefore that coordinative structures are best studied as ensembles of nonlinear coupled oscillators, exhibiting limit-cycle properties (Kugler et al., 1980; Turvey, 1990). The energy for sustaining cyclical motion was hypothesized to be regulated using information in an autonomous fashion. Indeed, cyclical limb movement has been shown to exhibit limitcycle properties, and coordinated rhythmic limb movement has been modeled successfully as a system of coupled nonlinear oscillators (e.g., see Haken, Kelso, \& Bunz, 1985; Kay, Kelso, Saltzman, \& Schöner, 1987; Kelso et al., 1981). As a result of these developments, dynamic systems theory has had an enormous impact on human movement science (for reviews, see Beek, Peper, \& Stegeman, 1995; Haken, 1996; Kelso, 1995; Kelso, DelColle, \&

Frank T. J. M. Zaal and Geoffrey P. Bingham, Psychology Department, Indiana University Bloomington; Richard C. Schmidt, Psychology Department, College of the Holy Cross.

We thank Betty Tuller and Claudia Carello for helpful comments on an earlier version of this article.

Correspondence concerning this article should be addressed to Frank T. J. M. Zaal, who is now at the Faculty of Human Movement Sciences, Vrije Universiteit, Van der Boechorststraat 9, 1081 BT Amsterdam, the Netherlands. Electronic mail may be sent to fzaal@fbw.vu.nl.
Schöner, 1990; Schmidt \& Turvey, 1995; Turvey, 1990). However, a brief review of this research reveals that the perceptual variables used in coordinated limb movement are not yet well understood, although the prominent role of perceptual information is widely recognized. Coordination during rhythmic limb movements has been measured and described in terms of the relative phase of the limbs. The amount of fluctuation in relative phase reflects the stability of coordination. We set out to study the perception of relative phase and phase variability. We suggest that relative phase and phase variability are perceptible properties and that their relative salience contributes to patterns of stability and instability in rhythmic limb movements. If this turns out to be true, then an investigation of how phase is perceived will significantly extend our understanding of coordination. For the present, we investigate relative visual sensitivity to these two variables and the stability of their perception, comparing our results to results from movement studies.

In the coordination of two rhythmically moving limbs, two patterns of relative phasing of the two limbs are characteristically more stable than others. Although people are able to learn other patterns with concerted practice (Schöner, Zanone, \& Kelso, 1992; Zanone \& Kelso, 1992), generally speaking, people move either in an in-phase pattern, in which the limbs move in a symmetrical fashion, or in an antiphase pattern, in which the limbs move in an alternating fashion (Kelso, 1984; Kelso, 1995; Kelso et al., 1981; Kelso, Schöner, Scholz, \& Haken, 1987; Schmidt, Shaw, \& Turvey, 1993; Tuller \& Kelso, 1989; Turvey, Rosenblum, Schmidt, \& Kugler, 1986; Wimmers, Beek, \& van Wieringen, 1992; Yamanishi, Kawato, \& Suzuki, 1980). Furthermore, the in-phase pattern has been demonstrated to be more stable than the antiphase pattern. The differential stability of these relative phases (and the stability of all other relative phases) is a function of the common frequency and of differences in eigenfrequency of the oscillators, among 
other things. ${ }^{1}$ With increasing frequency, the antiphase pattern becomes progressively less stable. At some critical frequency, the stability of the antiphase pattern vanishes, leaving only the inphase pattern as stable. The differential stability of the in-phase and antiphase patterns has been shown in experiments in which participants were instructed to increase or decrease the (common) frequency of movement during a trial. When participants are instructed to start moving in an antiphase pattern, to move gradually faster and faster, and not to resist the urge to switch patterns, a transition from the antiphase to an in-phase pattern occurs (e.g., Kelso, 1984; Kelso et al., 1981; Kelso et al., 1987; Schmidt, Carello, \& Turvey, 1990; Scholz \& Kelso, 1989). In contrast, starting in an in-phase pattern does not lead to a transition. At movement frequencies lower than the transition frequency, the difference in stability is revealed by larger fluctuations in the phasing of the two limbs in the antiphase mode (Schöner, Haken, \& Kelso, 1986). The transition from the antiphase pattern to the in-phase pattern exhibits the signature of a second-order nonequilibrium phase transition; that is, critical fluctuations and critical slowing down are observed as the transition frequency is approached (Kelso, Scholz, \& Schöner, 1986; Scholz \& Kelso, 1989; Schöner et al., 1986). More and larger departures from antiphase are observed, and any departure lasts longer, so that the limb takes longer to return to antiphase.

Differences in eigenfrequency between the two oscillating limbs also affect the stability properties of the in-phase and antiphase pattern (the eigenfrequency is the preferred frequency at which the limb will be oscillated by itself, that is, when not being coordinated with another limb). Both the relative phase actually produced (as opposed to the intended relative phase in accord with instructions) and the amount of variability in relative phase are functions of the size of the difference in eigenfrequencies (Kelso \& Jeka, 1992; Kugler \& Turvey, 1987; Schmidt et al., 1993; Schmidt \& Turvey, 1995; Turvey, Schmidt, \& Beek, 1993). The observed mean relative phase differs from perfect in-phase or antiphase if the eigenfrequencies of the two oscillators are different. The larger the difference in eigenfrequencies, the larger is the deviation from perfect in-phase or antiphase movement. These deviations in mean relative phase are accompanied by increases in phase variability.

The stability properties of the in-phase and antiphase patterns have been modeled in terms of a potential function. First formulated by Haken et al. (1985) to capture the transition phenomena under frequency scaling, and later extended to include a stochastic term (Kelso et al., 1987) and to account for differences in eigenfrequency (Kelso et al., 1990; Kelso \& Jeka, 1992), the HakenKelso-Bunz model reads

$$
V(\phi)=-\Delta \omega \phi-a \cos (\phi)-b \cos (2 \phi)-\sqrt{Q} \xi_{t} \phi,
$$

in which potential $V$ is a function of relative phase $\phi$. The difference in eigenfrequencies $(\Delta \omega)$ enters into Equation 1 as the first term on the right-hand side, frequency is related to the ratio of the variables $a$ and $b$ in the second and third terms, and the last term represents a low-amplitude stochastic force (of size $\sqrt{Q} ; \xi_{t}$ is Gaussian noise of unit size). For certain parameter values, associated with low frequencies, the two stable patterns appear as wells in the potential function. Increase of frequency leads to a gradual elimination of the potential well associated with the antiphase pattern, such that beyond a critical frequency only the in-phase pattern is stable, and a transition to this mode is inevitable. As the potential well gradually becomes more shallow, fluctuations increase and the stability of the movement decreases.

In the research on interlimb coordination, relative phase has proven to be a good variable for capturing the overall behavior of the system, an order variable from the perspective of synergetics (e.g., Haken et al., 1985). However, as pointed out by Bingham, Schmidt, and Zaal (1999), in spite of its success as an observable, the role of relative phase in the organization and control of behavior has not been resolved. Not much is known about the information used in tasks such as bimanual coordination. Clearly, people are able to perceive relative phase at least well enough to be able to move their limbs either at $0^{\circ}$ or at $180^{\circ}$ relative phase when asked to do so. On the other hand, participants seem to be unaware of the deviations from perfect in-phase or antiphase movement that result from differences in eigenfrequency between the two oscillators.

Evidence that visual information about relative phase can be detected and used in interlimb coordination comes from experiments demonstrating that the various transition phenomena are present when the two moving limbs are those of two different people. Schmidt et al. (1990) asked two people to coordinate with one another while each oscillated a lower leg. In this situation, the coupling between the limbs was entirely visual. Nevertheless, all of the results of the original Kelso experiments were replicated. Similarly, the entire set of results was replicated by Wimmers et al. (1992), who asked a single person to coordinate his lower arm movement with a target oscillating in a visual display.

The fact that people are able to perceive relative phase in situations other than in bimanual coordination has been demonstrated in a number of studies that used visual tasks (e.g., Dijkstra, Schöner, \& Gielen, 1994; Dijkstra, Schöner, Giese, \& Gielen, 1994; Giese, Dijkstra, Schöner, \& Gielen, 1996), haptic tasks (e.g., Jeka, Oie, Schöner, Dijkstra, \& Henson, 1998; Jeka, Schöner, Dijkstra, Ribeiro, \& Lackner, 1997), and speech-related tasks (e.g., Tuller \& Kelso, 1989). Kelso and colleagues investigated the perception of relative phase in intralimb coordination (Haken, Kelso, Fuchs, \& Pandya, 1990; Kelso, 1990; Kelso \& Pandya, 1991). Participants observed stick figures of simulated cyclical arm movement. Mean relative phase between wrist and elbow movement ranged from $0^{\circ}$ to $180^{\circ}$, in steps of $30^{\circ}$. Observers were instructed to categorize the displayed movements as either inphase $\left(0^{\circ}\right)$ or antiphase $\left(180^{\circ}\right)$. As one might expect, this classification was done most reliably when displayed relative phase was close to one of the prototypes (i.e., $0^{\circ}$ or $180^{\circ}$ ) and was more variable when displayed relative phase was between the prototypes. These and other results (e.g., see also Bertenthal \& Pinto, 1993; Johansson, 1950/1994) suggest that relative phase is perceptible or, at least, that the in-phase and antiphase patterns of movement can be distinguished. However, these studies do not shed much light on the stability properties of relative phase qua visually perceived property (or, more generally, on the relevant informa-

\footnotetext{
${ }^{1}$ The stability of relative phase is affected by variables in addition to the frequency and the differences in eigenfrequency. Examples of such variables are handedness and attention (e.g., Amazeen, Amazeen, Treffner, \& Turvey, 1997; Riley, Amazeen, Amazeen, Treffner, \& Turvey, 1997; Treffner \& Turvey, 1995). Consideration of these variables is beyond the scope of the present article.
} 
tional properties in coordination of cyclical movement). Might fluctuations in relative phase also be perceptible? There is some evidence that suggests that they are.

The antiphase pattern becomes unstable at higher frequencies. Nevertheless, the transition to the in-phase mode is not inevitable. Recent studies have stressed the critical importance of the instruction not to intervene. These studies have shown that if people are not given the explicit instruction to allow the transition to happen, they are well able to perform antiphase patterns at high frequencies (Lee, Blandin, \& Proteau, 1996; Scholz \& Kelso, 1990; Zelaznik, Smith, Franz, \& Ho, 1997), although the variability of phasing does increase. We suggest that the transitions under a nonintervention instruction, because they are highly reproducible, should be attributed to the perception of a certain critical amount of phase fluctuation and a decision in accord with the instruction not to resist, not to correct, and to make a transition, letting the in-phase pattern take over. In this case, it is not the mean relative phase that needs to be perceived but the variability in relative phase. Alternatively, one might hypothesize that people are able to switch patterns by voluntarily manipulating the shape of the potential wells. An instruction to resist change would then lead to the strengthening of the attraction of the original movement pattern. This would imply that there exists a natural shape of the potential landscape, which can be changed voluntarily to concur with task instructions. Although this scenario would certainly be an explanation of the capability of people to resist the transition from the less stable pattern to the more stable pattern, there is no evidence that people are actually able to change the potential wells. Also, this would provide no account for the ability of participants to comply with the instruction to move specifically at either in-phase or antiphase or to recognize that a transition had occurred. An explanation in terms of the perception of phase variability would be more parsimonious. However, the two accounts need not be at odds. The notion of phase perception is not inconsistent with a dynamical account and, in fact, must be part of such an account. The informational nature of the coupling between the limbs is well recognized whether it be haptic/kinesthetic in the case of withinperson coordination or visual in the case of between-persons coordination. The dynamical approach itself mandates an investigation of the informational-that is, perceptual-component of the behaviors. Hopefully, the additional information obtained from such investigations will allow the formulation of more detailed models in the future.

Our objective in the current work is to investigate the perception of both relative phase and phase variability and to investigate the respective perceptual stability. As discussed above, the same phenomena were demonstrated both in a task involving within-subject bimanual coupling and in a task involving between-subjects bimanual coupling. In the latter case, vision might be attributed a critical role, whereas in the former case proprioception might be the most relevant modality. We chose to use a visual task to study the perception of relative phasing because of the ease of performing psychophysics in a visual task, which allowed great precision in the experiments. The results should be indicative of the relevant perceptible properties used to visually coordinate tasks like bimanual rhythmic movement.

This study builds on our recent research on the visual perception of relative phase in human movements (Bingham et al., 1999). In a series of psychophysical experiments, participants observed two circles oscillating on a computer screen. These circles denoted the outlines of spheres at the lower end of a set of pendulums swung by a person in the sagittal plane. If one looked at the person from the side, the spheres would move along a circular path, from one extreme angle to the other. Correspondingly, the displays would present two circles along curved paths (compare to Figure 1; note, however, that in this figure the paths of the circles are straight instead of curved). In another situation, one could look at the motion of the spheres while facing the person swinging the pendulums. On an image plane, the outlines of the spheres would grow and shrink in an oscillating fashion. Now, the displays would present two circles increasing and decreasing in size over time. In fact, in any actual situation, the visual perspective on the oscillating pendulums would involve both. A pure side-to-side view of the movement (i.e., movement parallel to the frontoparallel plane) would yield only a common motion component in the optical pattern, whereas a pure in-depth view of the movement would yield only a relative motion component in the optical flow (Jansson, Bergström, \& Epstein, 1994). Such pure cases would be rare. Generic viewing would involve both components of motion, that is, both common and relative optical motion. For the first experiment, the kinematics of the displayed movement were taken from an earlier movement study in which participants swung a pair of hand-held pendulums at either $0^{\circ}$ or $180^{\circ}$ relative phase (Schmidt et al., 1993). By manipulating the length and inertia of the pendulums, different levels of relative phase other than $0^{\circ}$ and $180^{\circ}$ and accompanying phase variability were generated. Participants in the subsequent Bingham et al. (1999) study were asked to judge the coordination of the displayed movement, with the proviso that the level of coordination was meant to denote the amount of phase variability in the displays. The result was that judgments of coordination varied less with the actual phase variability than with the absolute deviations of mean relative phase from in-phase and antiphase movement. The more the actual movement deviated from either an in-phase or an antiphase pattern, the less coordinated it was judged. So, although the task was to judge phase variability, the judgments were related to relative-phase differ-
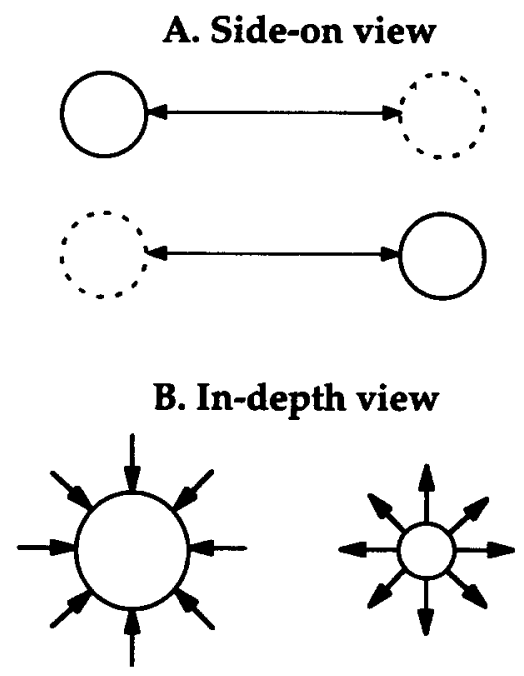

Figure 1. Schematic view of the displays in the side-on (A) and in-depth (B) viewing conditions. 
ences rather than phase variability in the displayed movement. A second finding was that the variability of judgments was higher if displayed movement was near an antiphase pattern than if it was near an in-phase pattern. Judgment variability also increased as relative phase deviated from either the in-phase or antiphase mode. Thus, the stability of judgments followed the pattern found in the interlimb coordination tasks.

In this first experiment, the kinematics were obtained from actual human pendulum-swinging movements. Because deviations in relative phase from the in-phase and antiphase modes tended to be accompanied by increases in phase variability, the relation between the coordination judgments and either mean relative phase or phase variability could not be studied independently. The second experiment reported in Bingham et al. (1999), therefore, used kinematics produced by a numerical simulation to generate the displays. In this way, relative-phase differences and phase variability could be manipulated independently. Once again, the judgments of phase variability covaried with mean relative phase more than with phase variability. Coordination patterns deviating from either in-phase or antiphase movement were judged as being more variable.

The finding that judgments of phase variability are affected by mean relative phase raises the question whether judgments of mean relative phase would be affected by phase variability and, if so, how. Apparently, observers do not visually perceive mean relative phase and phase variability independently in a task where they have to judge the latter. Thus, the information detected must be defined with respect to both mean relative phase and phase variability. Would the judgment of relative phase also be a function of both relative phase and phase variability? We investigated this by asking different sets of observers to judge either mean relative phase or phase variability. Observers in both cases judged the same set of displays, in which both mean relative phase and phase variability were manipulated. Displays were created using numerical simulations. This procedure allowed independent manipulation of relative phase and phase variability. We also used these judgments to investigate the stability of perception in each case. We investigated how the reliability of judgments of mean relative phase would vary with either mean relative phase or phase variability. Similarly, we investigated how the reliability of judgments of phase variability would vary. Finally, we investigated the effect on judgments of variation in the perspective from which the oscillation movements are viewed. Displayed movements were viewed by participants in four conditions. The four conditions were created by crossing two variables each with two levels. The first variable was the task, judging either mean relative phase or phase variability. The second variable was the visual perspective on the oscillatory events. In two of the conditions, two circles (referred to as balls below) moved from side to side on the computer screen, simulating movement in the frontoparallel plane (Figure 1A). In the other two conditions, movement toward and away from the observer was simulated, so that the circles were expanding and contracting on the screen (Figure 1B). These two viewing conditions are the limiting cases for possible orientations of straight ball trajectories viewed by an observer. They represent optical components combined in generic perspectives on such events. Second, by studying the psychophysics of relative phase perception and phase variability using displays involving totally different optical patterns with the same variation in the variables of interest (i.e., relative phase and phase variability), we aimed at ascertaining that the effects that we would observe were really to be attributed to the manipulation of those variables and not to properties of the particular visual perspective. In each viewing situation, participants were asked to judge mean relative phase in one condition and phase variability in another condition. A portion of the data, involving phase-variability judgments in the case where balls were moving from side to side, was described as the second experiment in Bingham et al. (1999).

\section{Method}

\section{Observers}

A different set of 10 observers, ranging in age from 18 to 46 years, participated in each of the four conditions. All observers had normal or corrected-to-normal vision and were free of motor disabilities. Observers in the condition in which phase variability had to be judged when balls were moving in depth participated in the study at Holy Cross and were not paid. Observers in other conditions participated in the study at Indiana University and were paid $\$ 7.50$ for participation. Each session lasted about $1.5 \mathrm{hr}$.

\section{Apparatus and Stimuli}

Two moving balls were simulated as two black line-drawn circles on a white background. They were presented on a Macintosh MO401 13-in. computer monitor with a $66.7-\mathrm{Hz}$ refresh rate. Every other frame was left blank, so that the effective presentation rate was $33.3 \mathrm{~Hz}$. The display was controlled by a Macintosh IIci computer. Participants viewed the displays from a distance of $70 \mathrm{~cm}$. The balls moved at a frequency of $1 \mathrm{~Hz}$. To eliminate reflections from the screen, we conducted the experiment in a darkened room.

The trajectories of the two balls were generated through numerical simulation. Two aspects of the relative motion of the two balls were manipulated. First, balls could move with a mean relative phase of $0^{\circ}, 30^{\circ}$, $60^{\circ}, 90^{\circ}, 120^{\circ}, 150^{\circ}$, or $180^{\circ}$. Second, at each level of relative phase, four levels of phase variability were determined in terms of standard deviations of relative phase equal to $0^{\circ}, 5^{\circ}, 10^{\circ}$, and $15^{\circ}$. Three instances of each combination of mean relative phase and phase variability were presented, yielding 84 trials per session. A single trial consisted of an 8-s display, followed by a screen displaying a computer-mouse-controlled slider. Observers were asked to enter their judgment by adjusting the slider in a range from 0 to 10 , with possible scores slightly smaller than 0 and slightly larger than 10 to remove any hard boundary at 0 and 10 , respectively.

Variability of relative phase was produced by slowing down and speeding up the individual oscillators. This was accomplished by manipulating the size of the time steps in the numerical simulations. A time step longer than a nominal time step (i.e., a time step appropriate for the display rate) would advance an oscillator, and a time step shorter than a nominal time step would delay an oscillator. By differentially changing the time steps of the two oscillators, their difference in phasing, hence their relative phase, was perturbed. The time steps were determined as follows. The time $t$ of each oscillator $i$ at time step $n$ was the time at the previous time step plus a modified (shortened or lengthened) new time step:

$$
t_{i}(n)=t_{i}(n-1)+\left(1+N_{i}^{*}\right) \delta t
$$

where $\delta t$ is the nominal time step of $0.03 \mathrm{~s}$. The temporal noise $N_{i}^{*}$ had two components:

$$
\begin{gathered}
N_{i}=A_{N, i} \cos \left(\omega_{\mathrm{N}} t\right)+0.1 A_{N, i} \xi_{n} \\
N_{i}^{*}=\left[-0.95<N_{i}<0.95\right] .
\end{gathered}
$$


First, it consisted of an oscillating component with a frequency $\omega_{N}$ of 1 , 0.5 , or 0.25 times the frequency of ball oscillation $\left(\omega_{\mathrm{B}}=1 \mathrm{~Hz}\right)$. This component had an amplitude $A_{N, i}$ that when combined with a smaller Gaussian component was appropriate to introduce a specific relative-phase difference between the two oscillators, that is, an amplitude such that over the entire trial the standard deviation of relative phase was $0^{\circ}, 5^{\circ}, 10^{\circ}$, or $15^{\circ}$. The oscillating component was combined with a smaller Gaussian component $\left(\xi_{t}\right.$ is Gaussian white noise of unit variance), with the restriction that the total advance or delay in timing of the oscillator was smaller than 0.95 times a nominal time step. The phase $\phi_{i}(n)$ of each oscillator at each time step then was

$$
\phi_{i}(n)=\omega_{\mathrm{B}} t_{i}(n)+\Delta \phi_{i}
$$

where $\Delta \phi_{i}$ is an initial phase offset to introduce differences in mean relative phase. Finally, the motion of each oscillator was generated as

$$
X_{i}(n)=A_{b} \cos \left(\phi_{i}(n)\right),
$$

where $A_{\mathrm{b}}$ is the amplitude of the ball motion.

In producing each level of variability in relative phase (standard deviation of $0^{\circ}, 5^{\circ}, 10^{\circ}$, or $15^{\circ}$ ), we added the noise to the oscillators in one of three different ways to ensure that phase variability was not confounded with specific kinematic characteristics, such as the timing of the end points in the oscillation. As a first method, noise signals of equal amplitude and opposite phase were added to each oscillator. A second method was to add noise signals with equivalent phase but with one amplitude triple the other. Third, a noise signal could be added to only one of the oscillators. We used a constrained random procedure to determine which oscillator received the larger perturbation in the second and third methods, so that each received it equally often.

The displays were generated from the numerically simulated kinematics in two ways. In two conditions, ball movement was parallel to the screen, moving side to side on the computer screen (side-on viewing; see Figure 1A). The size of the balls was $1.7 \mathrm{~cm}$, and their movement amplitude was $3.4 \mathrm{~cm}$. The path of the upper ball was $2.0 \mathrm{~cm}$ above the horizontal midline of the screen, and the path of the lower ball was $2.0 \mathrm{~cm}$ under this line. In the other two sessions, balls were simulated to be moving in depth (see Figure 1B). Here the size of the ball images expanded and contracted over a cycle. The maximum size was $2.5 \mathrm{~cm}$ and the minimum size was 1.3 $\mathrm{cm}$. The center of the left ball was $2.65 \mathrm{~cm}$ from the vertical midline of the computer screen, and the right ball's center was $2.65 \mathrm{~cm}$ to the right of this line.

\section{Procedure}

Each experimental session started with instructions and demonstrations. A series of examples illustrated both different mean relative phases and different levels of phase variability. The examples were accompanied by text explaining the manipulations and the task. Depending on the condition, observers were instructed to judge the mean relative phase or the phase variability in the displays. We explicitly explained that both mean relative phase and phase variability were manipulated but that just one of the two was to be judged. Next, several displays with samples of the possible manipulations, both mean relative phase and phase variability, were presented, together with the appropriate judgment score for the task. In the mean-relative-phase conditions, a score of 0 corresponded to movement at $0^{\circ}$, a score of 10 corresponded to movement at $180^{\circ}$, and scores in between 0 and 10 were to be given for mean relative phases between $0^{\circ}$ and $180^{\circ}$. In the phase-variability conditions, a score of 0 was to be given if no phase variability was present in the displayed movement. A score of 10 corresponded to the highest level of phase variability, as demonstrated in the exemplar trials. Observers had to read the description of the sample to be presented, watch the sample, and enter the appropriate score. A failure to enter the appropriate score led to a repeated presentation of the sample.
Furthermore, observers were invited to repeat each sample movement with accompanying explanation. They saw a minimum of 13 examples.

Following the initial practice session, each observer was presented with a blocked session, followed by a random session. The blocked session allowed for extensive training of the participants. It also enabled observers to concentrate on the aspect of the movements that they were to judge. For this reason, the organization of blocked trials was specific to the judgment task. If observers were to judge mean relative phase, trials were presented in four blocks, each of increasing phase variability, with relative phase randomized within a block. If observers were to judge phase variability, the blocked sessions consisted of seven blocks each of increasing mean relative phase (from $0^{\circ}$ to $180^{\circ}$ ) with the different levels of phase variability randomized within each block. In the following random sessions, all 84 displays were presented in a completely random order. No feedback on performance was provided during the blocked session and the random session.

The blocked sessions were intended to provide the participants with extensive training. Although patterns of judgments observed in the random sessions also occurred in the blocked sessions, we report analysis of the judgments in the random session only.

\section{Dependent Measures}

For each combination of mean relative phase and phase variability, we calculated the average judgment and the standard deviation over the three judgments for each participant. We used the former to study patterns of mean judgments and the latter to study the stability of judgments.

\section{Results}

We report the average judgment results by task. First, we present the results of the relative-phase judgments in both viewing conditions, followed by the phase-variability judgments in both viewing conditions. Finally, we compare the stability of the judgments across all conditions.

\section{Judging Mean Relative Phase}

Figure 2 presents the mean judgments when the balls were moving from side to side (Panel A) and when the balls were moving in depth (Panel B). Participants were well able to perceive the differences in mean relative phase. A repeated measures analysis of variance (ANOVA) with the between-subjects factor of viewing (side-on vs. in-depth) and within-subjects factors of phase $\left(0^{\circ}, 30^{\circ}, 60^{\circ}, 90^{\circ}, 120^{\circ}, 150^{\circ}\right.$, and $\left.180^{\circ}\right)$ and variability $\left(0^{\circ}, 5^{\circ}\right.$, $10^{\circ}$, and $\left.15^{\circ}\right)$ showed a significant phase effect, $F(6,108)=520.6$, $p<.001$. Both the Phase $\times$ Viewing interaction, $F(6,108)=5.4$, $p<.001$, and the Phase $\times$ Variability interaction, $F(18$, $324)=2.6, p<.001$, also reached significance. These interactions can be understood from the difference in the effect of adding variability in the $0^{\circ}$ relative-phase conditions between the viewing conditions. Adding variability did affect the judgments in the $0^{\circ}$ relative-phase conditions when balls were moving from side to side (see Figure $2 \mathrm{~A}$ ) but did not have an effect when balls were moving in depth (see Figure 2B). We performed separate ANOVAs on the mean judgments in the two viewing conditions, with factors of phase and variability, to demonstrate these effects. In the side-on viewing conditions, we found a significant phase effect, $F(6,54)=191.7, p<.001$, and a significant Phase $\times$ Variability interaction, $F(18,162)=2.6, p<.001$. We investigated this interaction by performing a simple effects analysis. This 

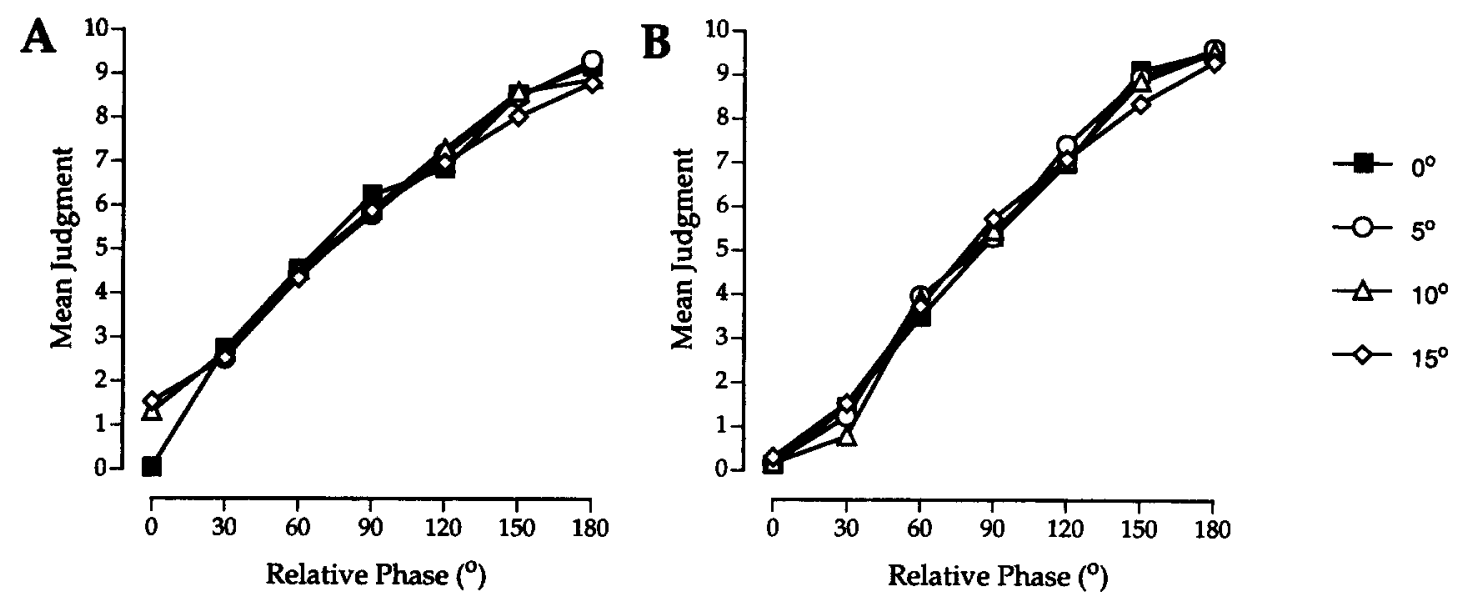

Figure 2. Average within-subject means of the mean-relative-phase judgments as a function of relative phase at the different phase-variability levels in the side-on (A) and in-depth (B) viewing conditions.

simple effects analysis indicated that the variability manipulations significantly affected the judgments in the $0^{\circ}, F(3,27)=13.7, p<$ .001 , and the $180^{\circ}$ (antiphase), $F(3,27)=3.3, p<.05$, conditions. As can be seen in Figure 2A, the more phase variability was added in the $0^{\circ}$ conditions, the more the judgments digressed from the correct score, indicating a $0^{\circ}$ relative phase. This effect was confirmed in a subsequent trend analysis of this simple effect, which showed a linear trend $F(1,9)=18.7, p<.005$. In contrast, the significant effect of added phase variability at $180^{\circ}$, as indicated by the simple effects analysis, did not have a systematic character according to the trend analysis.

The ANOVA on the mean judgments in the in-depth viewing conditions resulted only in a significant phase effect, $F(6,54)=$ $354.4, p<.001$. Again, participants clearly discriminated the different levels of phase difference present in the displays, but under these viewing conditions, added phase variability in the $0^{\circ}$ relative-phase conditions was not judged as a deviation from perfect in-phase movement.

\section{Judging Phase Variability}

Figure 3 presents the mean judgments as a function of the level of added phase variability in the side-on viewing condition (Panel A) and in the in-depth viewing condition (Panel B). Figure 4 presents the same mean judgments, now as a function of the actual mean relative phase, again in the side-on viewing condition (Panel A) and in the in-depth viewing condition (Panel B). As can be seen in Figure 3, participants' variability judgments were related to the levels of added phase variability only in the conditions in which mean relative phase was $0^{\circ}$, only when balls were moving from side to side (see Figure 3A), and not when balls were moving in depth. This resulted in a number of significant effects in a repeated measures ANOVA with the between-subjects factor of viewing (side-on vs. in-depth) and within-subjects factors of phase $\left(0^{\circ}, 30^{\circ}\right.$, $60^{\circ}, 90^{\circ}, 120^{\circ}, 150^{\circ}$, and $\left.180^{\circ}\right)$ and variability $\left(0^{\circ}, 5^{\circ}, 10^{\circ}\right.$, and $\left.15^{\circ}\right)$ on the mean judgments. Both the phase main effect, $F(6$, $108)=11.0, p<.001$, and the variability main effect, $F(3$,
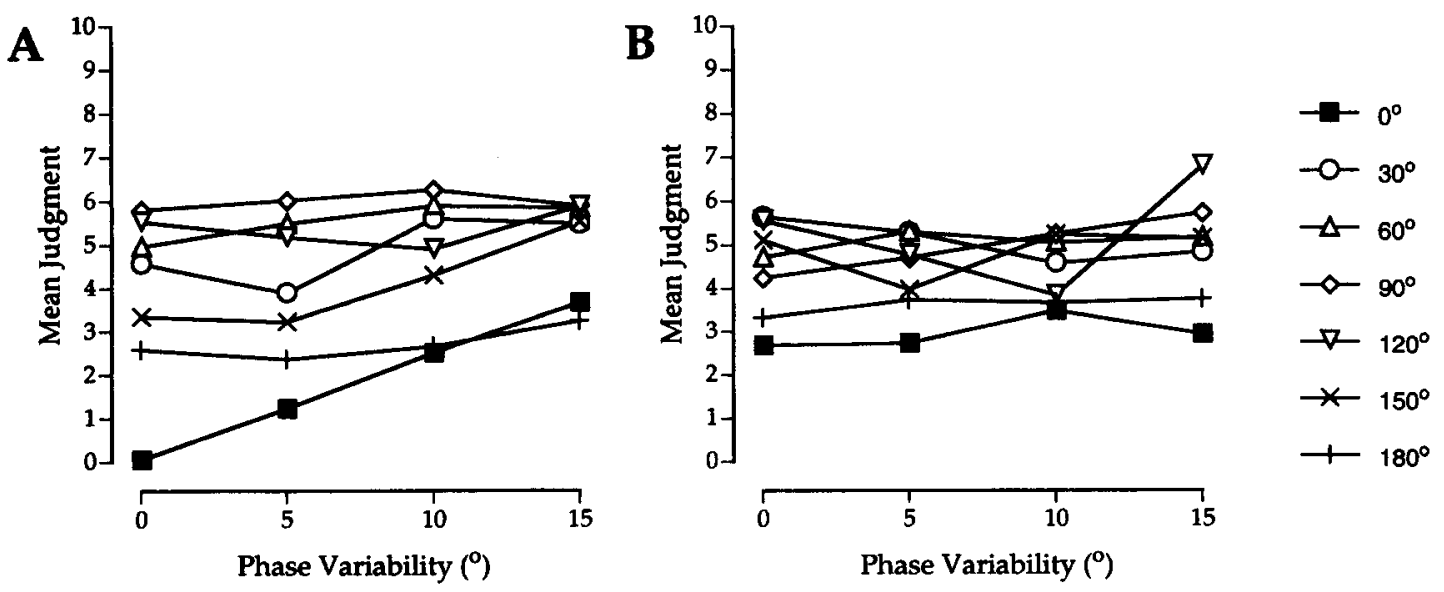

Figure 3. Average within-subject means of the phase-variability judgments as a function of phase variability at the different relative-phase levels in the side-on (A) and in-depth (B) viewing conditions. 

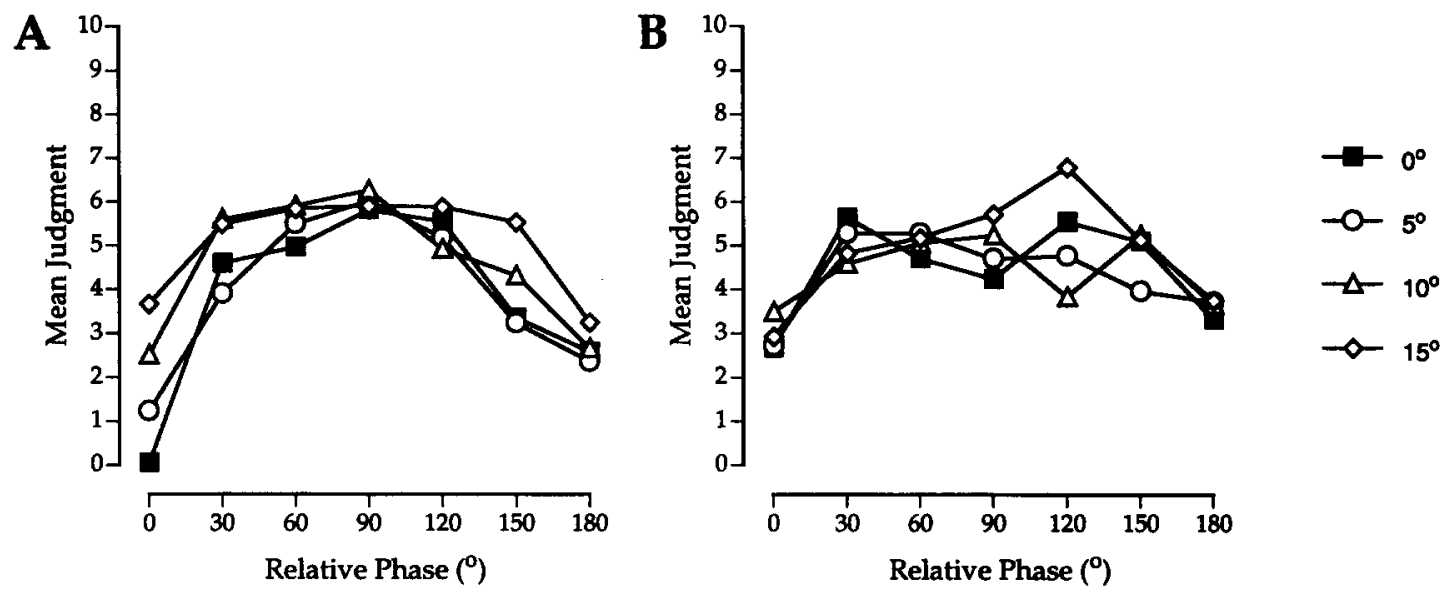

Figure 4. Average within-subject means of the phase-variability judgments as a function of relative phase at the different phase-variability levels in the side-on (A) and in-depth (B) viewing conditions.

54) $=11.7, p<.001$, were significant. In addition, the View $\times$ Variability interaction, $F(3,54)=3.0, p<.05$, the Phase $\times$ Variability interaction, $F(18,324)=2.6, p<.001$, and the View $\times$ Phase $\times$ Variability interaction, $F(18,324)=2.2, p<$ .005 , were also significant.

To investigate these interactions, we performed separate ANOVAs on the mean judgments for both viewing conditions. In the side-on viewing conditions, both the mean-phase manipulation and the phase-variability manipulation had a significant effect on the mean judgments. A repeated measures ANOVA, with factors of phase $\left(0^{\circ}, 30^{\circ}, 60^{\circ}, 90^{\circ}, 120^{\circ}, 150^{\circ}\right.$, and $\left.180^{\circ}\right)$ and variability $\left(0^{\circ}, 5^{\circ}, 10^{\circ}\right.$, and $\left.15^{\circ}\right)$ revealed a significant phase effect, $F(6$, 54) $=8.3, p<.001$, a significant variability effect, $F(3$, $27)=21.7, p<.001$, and a significant interaction, $F(18$, $162)=3.0, p<.001$. We first investigated the variability effect. As mentioned above, participants were not very well able to judge the added phase variability except for the condition in which mean relative phase was $0^{\circ}$ (see the filled squares in Figure 3A). To substantiate this observation, we performed simple effect tests on the mean judgments at each level of actual mean relative phase. These analyses yielded significant effects in the $0^{\circ}$ condition, $F(3$, $27)=14.1, p<.001$, in the $30^{\circ}$ condition, $F(3,27)=3.3, p<.05$, and in the $150^{\circ}$ condition, $F(3,27)=6.6, p<.005$. Figure $3 \mathrm{~A}$, however, shows that only the $0^{\circ}$ condition exhibited the correct ordering of mean judgments according to the different levels of phase variability.

The significant phase effect in the ANOVA resulted from an inverted U-shaped relation between the mean judgments and actual mean relative phase (see Figure 4A), as indicated by a significant quadratic trend, $F(1,9)=30.4, p<.001$. The linear trend did not reach significance. Note that although these were judgments of phase variability, they appeared to be more related to the mean relative phase. Even in the conditions where no phase variability was present, mean judgments were related to mean relative phase in an inverted $U$-shaped fashion as confirmed by a simple effects test, $F(6,54)=11.3, p<.001$, and a finding of quadratic, $F(1$, $9)=50.3, p<.001$, and cubic trends, $F(1,9)=7.8, p<.05$. Movement at $180^{\circ}$ mean relative phase was thus seen as more variable than $0^{\circ}$ movement, whereas movement at relative phases in between those extremes was seen as even more variable.

The phase-variability judgments of the participants who observed the movements in depth followed a similar but not identical pattern to that obtained when participants observed the movements from the side. In this case, the judgments were not significantly affected by the added phase variability. A repeated measures ANOVA on the judgments in the in-depth viewing condition revealed that the judgments of phase variability varied with mean relative phase, $F(6,54)=3.3, p<.01$, but not with the level of phase variability. A significant quadratic trend was present in the relation between actual relative phase and the judgments of phase variability, $F(1,9)=6.0, p<.05$, demonstrating the significance of the inverted U-shape of this relation, as shown in Figure 4B. The interaction between phase and variability was also significant, $F(18,162)=2.0, p<.05$. Simple effects analyses revealed significant effects of variability at relative phases of $120^{\circ}, F(3$, $27)=5.3, p<.01$, and of $150^{\circ}, F(18,162)=4.4, p<.05$. However, the order of the mean judgments did not follow a systematic relation with the level of phase variability.

As with the findings in the side-on viewing condition, the mean judgments in the conditions in which no phase variability was added were significantly affected by mean relative phase, as shown by a simple effects test, $F(6,54)=3.3, p<.01$, although in this case, the quadratic trend was just shy of the $p=.05$ level, $F(1$, 9) $=5.0, p=.052$.

\section{Comparison of the Judgment Stability Among Conditions}

We investigated the stability of the judgments under the different viewing conditions and different judgment tasks by comparing the standard deviations of the judgments. The standard deviations, averaged over observers, for the relative-phase judgments and for the phase-variability judgments, respectively, are presented in Figures 5 and 6 as a function of mean relative phase. Figure 7 presents the standard deviations for the phase-variability judgments as a function of the level of added phase variability. We performed ANOVAs on the standard deviations of all the judgments, with 

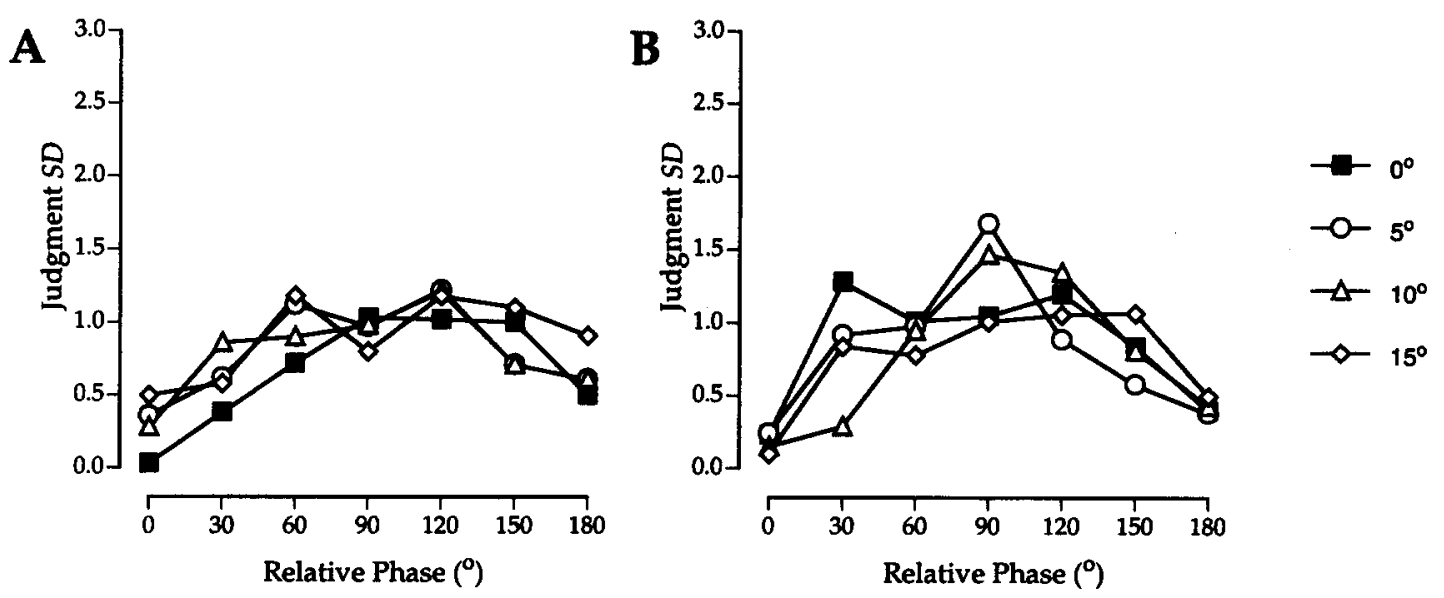

Figure 5. Average within-subject standard deviations of the mean-relative-phase judgments as a function of relative phase at the different phase-variability levels in the side-on (A) and in-depth (B) viewing conditions.

between-subjects factors of task (judging mean relative phase vs. phase variability) and viewing (side-on vs. in-depth) and withinsubject factors of phase $\left(0^{\circ}, 30^{\circ}, 60^{\circ}, 90^{\circ}, 120^{\circ}, 150^{\circ}\right.$, and $\left.180^{\circ}\right)$ and variability $\left(0^{\circ}, 5^{\circ}, 10^{\circ}\right.$, and $\left.15^{\circ}\right)$.

All four main effects in the ANOVA were significant: task, $F(1$, 36) $=93.8, p<.001$; viewing, $F(1,36)=4.8, p<.05$; phase, $F(6,216)=12.8, p<.001$; and variability, $F(1,36)=55.4, p<$ .001 . Also significant were the Task $\times$ Viewing interaction, $F(1$, $36)=4.2, p<.05$, and the Task $\times$ Viewing $\times$ Phase interaction, $F(6,216)=3.4, p<.005$. The variability main effect reflected decreasing judgment stabilities with increasing amounts of phase variability $(1.23,1.31,1.34$, and 1.44 at phase-variability levels of $0^{\circ}, 5^{\circ}, 10^{\circ}$, and $15^{\circ}$, respectively). Judgments of mean relative phase were more stable than judgments of phase variability (SDs of 0.80 and 1.86 , respectively). This difference in judgment standard deviations was greater in the in-depth viewing conditions (mean $S D$ s of 0.80 and 2.10 for the mean-relative-phase judgments and phase-variability judgments, respectively) than in the side-on viewing conditions (mean SDs of 0.79 and 1.63 in the mean- relative-phase judgments and phase-variability judgments, respectively). Note that there was a difference between the phasevariability-judgment standard deviations ( 2.10 vs. 1.63$)$ but not between the mean-relative-phase-judgment standard deviations ( 0.80 vs. 0.79 ).

The phase main effect reflected an inverted $U$-shaped relation between judgment standard deviations and actual relative phase (see Figures 5 and 6). The Task $\times$ Viewing $\times$ Phase interaction was due to the fact that in the in-depth condition, the inverted $\mathrm{U}$-shaped relation was only present in mean-relative-phase judgments and not in phase-variability judgments. The inverted $U$-shaped relation was present for both in the side-on viewing conditions. We performed separate ANOVAs (with factors of phase and variability) on the judgment standard deviations in the different viewing conditions and for the different tasks to strengthen this observation. These ANOVAs demonstrated the significance of the phase effects in both the situations in which participants judged mean relative phase, $F(6,54)=8.4, p<.001$ (side-on) and $F(6,54)=9.4, p<.001$ (in-depth), and the situation
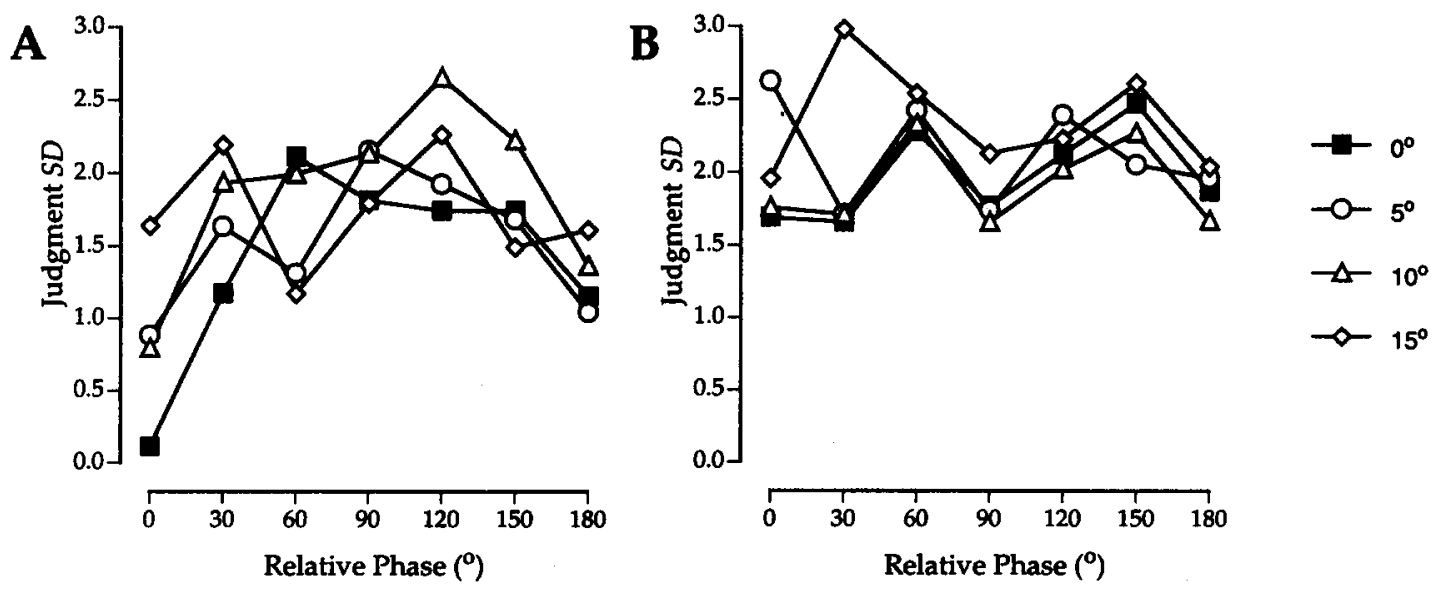

Figure 6. Average within-subject standard deviations of the phase-variability judgments as a function of relative phase at the different phase-variability levels in the side-on (A) and in-depth (B) viewing conditions. 

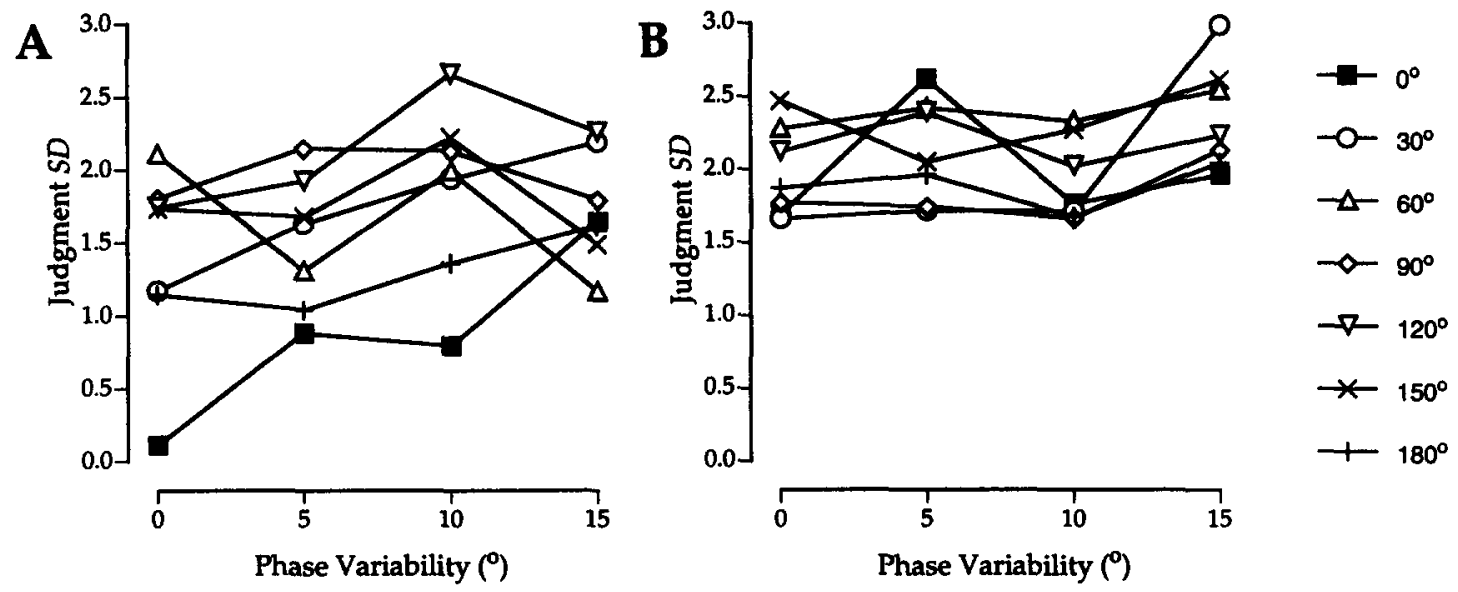

Figure 7. Average within-subject standard deviations of the phase-variability judgments as a function of phase variability at the different relative-phase levels in the side-on (A) and in-depth (B) viewing conditions.

in which participants judged phase variability and balls were moving from side to side, $F(6,54)=6.1, p<.001$. However, the ANOVA did not show a significant phase effect in the situation in which participants judged phase variability and balls were moving in depth. Trend analyses with respect to all three significant phase effects mentioned resulted in significant linear and quadratic trends in all cases: relative-phase judgments for side-on movement, $F(1,9)=15.0, p<.005$ (linear trend) and $F(1,9)=32.2$, $p<.001$ (quadratic trend); relative-phase judgments for in-depth movement, $F(1,9)=5.8, p<.05$ (linear trend) and $F(1$, 9 ) $=25.2, p<.001$ (quadratic trend); phase-variability judgments for side-on movement, $F(1,9)=11.2, p<.01$ (linear trend) and $F(1,9)=19.9, p<.005$ (quadratic trend). The linear trend substantiates the observation that judgment variability was higher at $180^{\circ}$ than at $0^{\circ}$, whereas the quadratic trend shows that judgment variability did increase when phase deviated from $0^{\circ}$ and $180^{\circ}$.

In both the situations in which participants judged mean relative phase, as well as in the situation in which participants judged phase variability and balls were moving from side to side, even in the conditions in which no phase variability was present, the stability of the judgments was affected by the level of mean relative phase. Simple effects analyses revealed significant phase effects: side-on viewing and relative-phase judgments, $F(6,54)=4.8, p<.001$; in-depth viewing and relative-phase judgments, $F(6,54)=3.3$, $p<.01$; and side-on viewing and phase-variability judgments, $F(6,54)=4.2, p<.005$. In the two side-on viewing conditions, trend analyses indicated linear trends-relative-phase judgments, $F(1,9)=40.8, p<.001$; phase-variability judgments, $F(1$, 9) $=10.2, p<.05$-as well as quadratic trends-relative-phase judgments, $F(1,9)=17.8, p<.005$; phase-variability judgments, $F(1,9)=15.4, p<.005$-whereas only a quadratic trend was significant in the in-depth viewing condition, $F(1,9)=22.4, p<$ .005 .

In summary, the variability of phase-variability judgments was higher, irrespective of viewing conditions. Side-on viewing led to more stable judgments of phase variability, but viewing condition did not have an effect on the judgment stability for judgments of mean relative phase. An inverted $U$-shaped relation between judg- ment stability and mean relative phase was found to be present in all data except the phase-variability judgments under in-depth viewing.

\section{Discussion}

Observers were well able to judge mean relative phase. Whether viewing side on or in depth, observers' mean judgments exhibited a linear relation with mean relative phase. Therefore, the fact that the situation with only two stable modes is observed in bimanual coordination studies cannot be attributed to a complete inability to perceive the intermediate relative phases per se. This finding is not too surprising given that people can oscillate their fingers at relative phases other than $0^{\circ}$ and $180^{\circ}$, as found by Tuller and Kelso (1989) and Yamanishi et al. (1980). Although participants in the Tuller and Kelso study were able to perform relative phases other than $0^{\circ}$ and $180^{\circ}$, the stability of those relative phases, as indexed by the standard deviations of relative phase, decreased with departures from $0^{\circ}$ and $180^{\circ}$. We replicated this "seagull effect" in three of the four conditions of the perceptual judgments. Standard deviations of judgments exhibited an inverted $U$-shaped function of relative phase. This occurred both for judgments of mean relative phase (in both viewing conditions) and judgments of phase variability (in the side-on viewing condition). When relative phase was at $0^{\circ}$, judgments were most stable. When relative phase was at $180^{\circ}$, judgments were less stable, and when relative phase was between $0^{\circ}$ and $180^{\circ}$, the stability of judgments decreased as a function of the departure from $0^{\circ}$ and $180^{\circ}$. Note that the same pattern of stability was seen for both tasks, involving different types of judgments. The inverse $U$-shape of the relation between judgment stability and mean relative phase parallels the shape of the potential function in the Haken-Kelso-Bunz model (Haken et al., 1985), as exemplified in Figure 8. We fitted Equation 1, without the Gaussian noise term, to the standard deviations of the relative-phase judgments at phase-variability levels of $0^{\circ}$ in the side-on viewing condition (compare Figure 8 to Figure 5A).

Our results demonstrate that mean relative phase and phase variability are not independent as perceptible properties of coordinated motion. First, the mean-relative-phase judgments were 


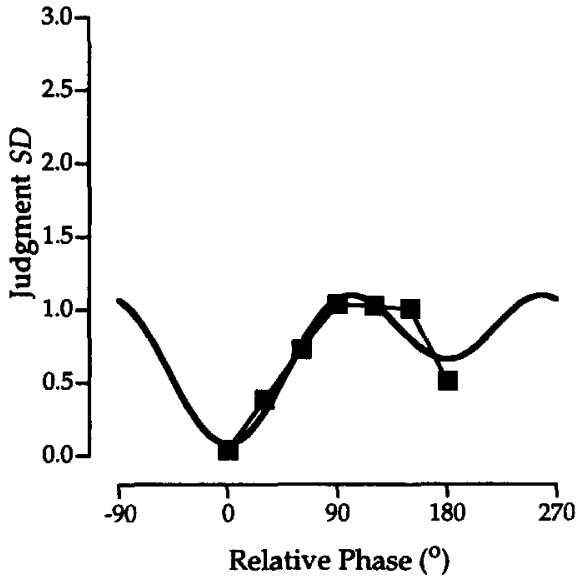

Figure 8. Potential functions (solid lines), obtained by fitting the HakenKelso-Bunz model (Equation 1) to the mean-relative-phase-judgment standard deviations at the $0^{\circ}$ phase-variability levels in the side-on viewing condition as a function of mean relative phase and these judgment standard deviations.

affected by the amount of phase variability. Adding phase variability resulted in systematic deviations of the mean-relative-phase judgments in the $0^{\circ}$ condition. In that case, movements having phase variability were judged as at $10^{\circ}$ to $30^{\circ}$ relative phase, depending on the amount of variability. An interaction of mean relative phase and phase variability was present even more clearly in the phase-variability experiments. Again, the $0^{\circ}$ relative-phase condition seemed to have a special status in that only in these conditions did observers systematically show a sensitivity to the phase-variability levels in the displayed movements. As soon as the mean-relative-phase deviated from $0^{\circ}$, no systematic relation between the phase-variability judgments and the added phase variability could be established. Most significantly, the judgments of phase variability were best predicted by the mean relative phase in the displayed movement, replicating the results found in Bingham et al. (1999). The relation between the judgments and mean relative phase took the form of an asymmetric inverted $U$, with antiphase movement being judged more variable than in-phase movement. Deviations from the two stable modes were seen as being more variable, even if the movement contained no phase variability. This last fact is most revealing. A $90^{\circ}$ mean relative phase was judged as highly variable even when the movement contained no phase variability.

The fact that observers' phase-variability judgments covaried with mean relative phase rather than with phase variability cannot be attributed to a lack of understanding by participants of the difference between relative phase and phase variability or to a failure to understand the task. This is shown by the phasevariability judgments in the $0^{\circ}$ mean-relative-phase condition in which balls were moving from side to side. A linear relation was exhibited between the phase-variability judgments and the level of phase variability. Clearly, when phase variability was a salient property of the displayed movement, observers' judgments were related to the level of phase variability.

The results of the perceptual judgment study presented here, together with those reported in Bingham et al. (1999), are similar to several findings in bimanual coordination studies. How should these results be interpreted with respect to the dynamical systems perspective on perception-action? This perspective has provided the theoretical basis for the discovery, understanding, and continued investigation of these phenomena. There are at least two ways that the current results might be interpreted in this perspective. First, one could argue that the patterns of coordinated rhythmic limb movements are physically constrained to obey the lawful relations of coupled nonlinear oscillators, and because of this, perception of relative phase and phase variability is tuned to the same relations, and the same pattern of results will thus be found in studies of perception. In the case of bimanual coordination, the Haken-Kelso-Bunz model (Haken et al., 1985) predicts a relation between mean relative phase and phase variability. The potential function in this model reflects the stability of the system and hence the variability along the relative-phase continuum. This function predicts that variability increases when relative phase deviates from an in-phase or an antiphase mode. Variability is highest around $90^{\circ}$ mean relative phase. Also, according to this model, antiphase movement is more variable than in-phase movement (i.e., the potential well at $0^{\circ}$ is deeper than that at $180^{\circ}$ ). In addition, eigenfrequency differences between the two oscillating limbs lead to changes in the relation between relative phase and phase variability. If the limbs have different eigenfrequencies, then the stable relative phases are slightly different from $0^{\circ}$ and $180^{\circ}$, and the phase variability associated with each relative phase is different. Given these facts (and the assumption that they are physically determined), then one can argue that that perception would also exhibit these patterns because it is attuned to action.

A second interpretation of the results follows from the assumption that patterns of coordinated rhythmic movement are generated by and, thus, ultimately reflect perceptual abilities. In this case, the phenomena would reflect the ability to perceive relative phase and phase variability. Our results demonstrate that relative phases away from the two stable modes, $0^{\circ}$ and $180^{\circ}$, are perceived as less stable. Even though movement at $90^{\circ}$ may be perfectly stable, it is not seen as such. This suggests that, if asked to produce stable movement at $90^{\circ}$, a person could not do so simply because he or she could not perceive it. One cannot stably control what one cannot perceive. Phase variability led observers in our experiments to judge movement that was in phase on average as deviating from in-phase movement. This latter result might provide some understanding of deviations of mean phase from $0^{\circ}$ and $180^{\circ}$ that have been found when participants are required to oscillate together two pendulums that have different lengths and thus different eigenfrequencies. If the eigenfrequency difference yields greater phase variability, then oscillation at $30^{\circ}$ could not be distinguished from oscillation at $0^{\circ}$. Of course, this presumes and does not account for the increase in phase variability, as produced by a difference in eigenfrequency of the individual oscillators.

Although these might seem to be contrasting viewpoints, we see the two interpretations as complementary rather than opposing. On the action side, research has made it increasingly clear that coherent, coordinated action is not possible without perception. This is well illustrated by the work of Cole (1995), for instance, and related work by Ghez and colleagues (e.g., Ghez, Gordon, Ghilardi, \& Sainburg, 1995) and is consistent with prevailing mass-spring models of limb movement that incorporate sensory components into the functional mass-spring organization (Feld- 
man, 1986; Feldman, Adamovich, Ostry, \& Flanagan, 1990; Hogan, 1985; Hogan, Bizzi, Mussa-Ivaldi, \& Flash, 1987). In fact, proponents of a dynamical approach to perception-action describe information as an inherent component of movement dynamics, that is, as part of the physical determination of movement patterns (e.g., see Kelso, 1995, and references therein). The problem is to reveal the exact nature of perception's role and, relatedly, the form of the information that is used. It is to this problem that we have directed our efforts. The question provoked by the current results is how is relative phase apprehended? Two possibilities are suggested by measures of relative phase that have been used in movement studies. A continuous-phase measure tracks the relative phase at all points along the movement trajectory. The measure incorporates both the positions and the velocities of each oscillator along its trajectory. In contrast, a point-phase measure uses only the positions at times that the oscillators are at zero velocity, that is, at the end points of the movement. Alternatively, positions at times of peak velocity could be used. The question is whether the perception of relative phase is more similar to a continuous-phase or a point-phase measure. The fact that mean relative phases of $0^{\circ}$ and $180^{\circ}$ are better resolved, and $0^{\circ}$ better than $180^{\circ}$, suggests that perception might resemble a point-phase measure. If the relative positions at the end points or peak velocity of the movement are the key, then they would be apprehended more readily when they occur at the same time and phase. This would be consistent with several suggestions in the literature that limb oscillations are anchored to the endpoints of the oscillation. These suggestions were inspired by the observation that the phase variability around the endpoints of movement is lower. This would be expected to occur at the point in a cycle where discrete information about the prescribed timing is used (Byblow, Carson, \& Goodman, 1994; Byblow, Chua, \& Goodman, 1994; Michaels \& Bongers, 1994; see also Beek, 1989). On the other hand, the relative ability to resolve positions at high velocity together with the ability to resolve velocity differences at a Weber ratio of about $5 \%$ (McKee, 1981) might also account for the current results in the context of a continuous-phase measure. These issues remain for future research. Their resolution may provide the foundation for revised dynamical models of coordinated rhythmic movement, models that include perceptual variables explicitly. The current studies establish both the relevance of perception for understanding the human movement results and the need for additional perceptual studies in this regard.

\section{References}

Amazeen, E. L., Amazeen, P. G., Treffner, P. J., \& Turvey, M. T. (1997). Attention and handedness in bimanual coordination dynamics. Journal of Experimental Psychology: Human Perception and Performance, 23, 1552-1560.

Beek, P. J. (1989). Juggling dynamics. Amsterdam: Vrije Universiteit Press.

Beek, P. J., Peper, C. E., \& Stegeman, D. F. (1995). Dynamical models of movement coordination. Human Movement Science, 14, 573-608.

Bernstein, N. A. (1967). The coordination and regulation of movements. Oxford, UK: Pergamon Press.

Bertenthal, B. I., \& Pinto, J. (1993). Complementary processes in the perception and production of human movements. In L. B. Smith \& $\mathbf{E}$. Thelen (Eds.), A dynamic systems approach to development: Applications (pp. 209-239). Cambridge, MA: MTT Press.
Bingham, G. P., Schmidt, R. C., \& Zaal, F. T. J. M. (1999). Visual perception of relative phasing of human limb movements. Perception \& Psychophysics, 61, 246-258.

Byblow, W. D., Carson, R. G., \& Goodman, D. (1994). Expressions of assymetries and anchoring in bimanual coordination. Human Movement Science, 13, 3-28.

Byblow, W. D., Chua, R., \& Goodman, D. (1994). Assymmetries in coupling dynamics of perception and action. Joumal of Motor Behavior, 27, 123-137.

Cole, J. (1995). Pride and the daily marathon. Cambridge, MA: MIT Press.

Dijkstra, T. M. H., Schöner, G., \& Gielen, C. C. A. M. (1994). Temporal stability of the action-perception cycle for postural control in a moving visual environment. Experimental Brain Research, 97, 477-486.

Dijkstra, T. M. H., Schöner, G., Giese, M. A., \& Gielen, C. C. A. M. (1994). Frequency dependence of the action-perception cycle for postural control in a moving visual environment: Relative phase dynamics. Biological Cybernetics, 71, 489-501.

Feldman, A. G. (1986). Once more on the equilibrium-point hypothesis for motor control. Joumal of Motor Behavior, 18, 17-54.

Feldman, A. G., Adamovich, S. V., Ostry, D. J., \& Flanagan, J. R. (1990). The origin of electromyograms-explanations based on the equilibrium point hypothesis. In J. M. Winters, \& S. L.-Y. Woo (Eds.), Multiple muscle systems: Biomechanics and movement organization (pp. 195213). Berlin: Springer Verlag.

Ghez, C., Gordon, J., Ghilardi, M. F., \& Sainburg. R. (1995). Contributions of vision and proprioception to accuracy of limb movements. In $\mathbf{M}$. Gazzaniga (Ed.), The cognitive neurosciences (pp. 548-564). Cambridge, MA: MTT Press.

Giese, M. A., Dijkstra, T. M. H., Schð̈ner, G., \& Gielen, C. C. A. M. (1996). Identification of the nonlinear state-space dynamics of the action-perception cycle for visually induced postural sway. Biological Cybernetics, 74, 427-437.

Haken, H. (1996). Principles of brain functioning: A synergetic approach to brain activity, behavior and cognition. Berlin: Springer Verlag.

Haken, H., Kelso, J. A. S., \& Bunz, H. (1985). A theoretical model of phase transitions in human hand movements. Biological Cybernetics, 51, 347356.

Haken, H., Kelso, J. A. S., Fuchs, A., \& Pandya, A. S. (1990). Dynamic pattern recognition of coordinated biological motion. Neural Networks, 3, 395-401.

Hogan, N. (1985). The mechanics of multijoint posture and movement control. Biological Cybemetics, 52, 315-331.

Hogan, N., Bizzi, E., Mussa-Ivaldi, F. A., \& Flash, T. (1987). Controlling multijoint motor behavior. Exercise and Sport Sciences Reviews, 15, 153-190.

Jansson, G., Bergström, S. S., \& Epstein, W. (Eds.). (1994). Perceiving events and objects. Hillsdale, NJ: Erlbaum.

Jeka, J., Oie, K., Schöner, G., Dijkstra, T., \& Henson, E. (1998), Position and velocity coupling of postural sway to somatosensory drive. Journal of Neurophysiology, 79, 1661-1674.

Jeka, J. J., Schöner, G., Dijkstra, T., Ribeiro, P., \& Lackner, J. R. (1997). Coupling of fingertip somatosensory information to head and body sway. Experimental Brain Research, 113, 475-483.

Johansson, G. (1994). Configurations in event perception. In G. Jansson, S. S. Bergstrorm, \& W. Epstein (Eds.), Perceiving events and objects (pp. 29-122). Hillsdale, NJ: Erlbaum. (Original work published 1994)

Kay, B. A., Kelso, J. A. S., Saltzman, E. L., \& Schöner, G. (1987). Space-time behavior of single and bimanual rhythmical movements: Data and limit cycle model. Journal of Experimental Psychology: Human Perception and Performance, 13, 178-192.

Kelso, J. A. S. (1984). Phase transitions and critical behavior in human bimanual coordination. American Journal of Physiology: Regulatory, Integrative and Comparative Physiology, 15, R1000-R1004.

Kelso, J. A. S. (1990). Phase transitions: Foundations of behavior. In H. 
Haken \& M. Stadler (Eds.), Synergetics of cognition (pp. 249-268). Berlin: Springer Verlag.

Kelso, J. A. S. (1995). Dynamic patterns: The self-organization of brain and behavior. Cambridge, MA: MIT Press.

Kelso, J. A. S., DelColle, J. D., \& Schöner, G. (1990). Action-perception as a pattern formation process. In M. Jeannerod (Ed.), Attention and performance XIII (pp. 139-169). Hillsdale, NJ: Erlbaum.

Kelso, J. A. S., Holt, K., Rubin, P., \& Kugler, P. N. (1981). Patterns of human interlimb coordination emerge from the properties of non-linear, limit cycle oscillatory processes: Theory and data. Journal of Motor Behavior, 13, 226-261.

Kelso, J. A. S., \& Jeka, J. J. (1992). Symmetry breaking dynamics of human interlimb coordination. Journal of Experimental Psychology: Human Perception and Performance, 18, 645-668.

Kelso, J. A. S., \& Pandya, A. S. (1991). Dynamic pattern generation and recognition. In N. I. Badler, B. A. Barsky, \& D. Zeltzer (Eds.), Making them move: Mechanics, control, and animation of articulated figures (pp. 171-190). San Mateo, CA: Morgan Kaufmann.

Kelso, J. A. S., Scholz, J. P., \& Schöner, G. (1986). Nonequilibrium phase transitions in coordinated biological motion: Critical fluctuations. Physics Letters A, 118, 279-284.

Kelso, J. A. S., Schöner, G., Scholz, J. P., \& Haken, H. (1987). Phaselocked modes, phase transitions and component oscillators in biological motion. Physica Scripta, 35, 79-87.

Kugler, P. N., Kelso, J. A. S., \& Turvey, M. T. (1980). On the concept of coordinative structures as dissipative structures: I. Theoretical lines of convergence. In G. E. Stelmach \& J. Requin (Eds.), Tutorials in motor behavior (pp. 3-47). Amsterdam: North-Holland.

Kugler, P. N., \& Turvey, M. T. (1987). Information, natural law and the self-assembly of rhythmic movement. Hillsdale, $\mathrm{NJ}$ : Erlbaum.

Lee, T. D., Blandin, Y., \& Proteau, L. (1996). Effects of task instructions and oscillation frequency on bimanual coordination. Psychological Research, 59, 100-106.

McKee, S. P. (1981). A local mechanism for differential velocity detection. Vision Research, 21, 491-500.

Michaels, C. F., \& Bongers, R. M. (1994). The dependence of discrete movements on rhythmic movements: Simple RT during oscillatory tracking. Human Movement Science, 13, 473-493.

Riley, A. R., Amazeen, E. L., Amazeen, P. G., Treffner, P. J., \& Turvey, M. T. (1997). Effects of temporal scaling and attention on the asymmetrical dynamics of bimanual coordination. Motor Control, 1, 263283.

Schmidt, R. C., Carello, C., \& Turvey, M. T. (1990). Phase transitions and critical fluctuations in the visual coordination of rhythmic movements between people. Journal of Experimental Psychology: Human Perception and Performance, 16, 227-247.

Schmidt, R. C., Shaw, B. K., \& Turvey, M. T. (1993). Coupling dynamics in interlimb coordination. Journal of Experimental Psychology: Human Perception and Performance, 19, 397-415.

Schmidt, R. C., \& Turvey, M. T. (1995). Models of interlimb coordination-equilibria, local analyses, and spectral patterning: Comment on
Fuchs and Kelso (1994). Journal of Experimental Psychology: Human Perception and Performance, 21, 432-443.

Scholz, J. P., \& Kelso, J. A. S. (1989). A quantitative approach to understanding the formation and change of coordinated movement patterns. Journal of Motor Behavior, 21, 122-144.

Scholz, J. P., \& Kelso, J. A. S. (1990). Intentional switching between patterns of bimanual coordination depends on the intrinsic dynamics of the patterns. Journal of Motor Behavior, 22, 98-124.

Schöner, G., Haken, H., \& Kelso, J. A. S. (1986). A stochastic theory of phase transitions in human hand movement. Biological Cybernetics, 53 , 247-257.

Schöner, G., Zanone, P. G., \& Kelso, J. A. S. (1992). Learning as change of coordination dynamics: Theory and experiment. Journal of Motor Behavior, 24, 29-48.

Treffner, P. J., \& Turvey, M. T. (1995). Handedness and asymmetric dynamics of bimanual rhthmic coordination. Journal of Experimental Psychology: Human Perception and Performance, 21, 318-333.

Tuller, B., \& Kelso, J. A. S. (1989). Environmentally specified patterns of movement coordination in normal and split-brain subjects. Experimental Brain Research, 75, 306-316.

Turvey, M. T. (1990). Coordination. American Psychologist, 45, 938-953.

Turvey, M. T., Rosenblum, L. D., Schmidt, R. C., \& Kugler, P. N. (1986). Fluctuations and phase symmetry in coordinated rhythmic movements. Journal of Experimental Psychology: Human Perception and Performance, 12, 564-583.

Turvey, M. T., Schmidt, R. C., \& Beek, P. J. (1993). Fluctuations in interlimb rhythmic coordination. In K. M. Newell \& D. M. Corcos (Eds.), Variability and motor control (pp. 381-411). Champaign, $\mathbb{L}$ : Human Kinetics Publishers.

Wimmers, R. H., Beek, P. J., \& van Wieringen, P. C. W. (1992). Phase transitions in rhythmic tracking movements: A case of unilateral coupling. Human Movement Science, 11, 217-226.

Yamanishi, J., Kawato, M., \& Suzuki, R. (1979). Studies on human finger tapping neural networks by phase transition curves. Biological Cybernetics, 33, 199-208.

Yamanishi, J., Kawato, M., \& Suzuki, R. (1980). Two coupled oscillators as a model for the coordinated finger tapping by both hands. Biological Cybernetics, 37, 219-225.

Yates, F. E. (1982). Outline of a physical theory of physiological systems. Canadian Journal of Physiology and Pharmacology, 60, 217-248.

Zanone, P. G., \& Kelso, J. A. S. (1992). Evolution of behavioral attractors with learning: Nonequilibrium phase transitions. Journal of Experimental Psychology: Human Perception and Performance, 18, 403-421.

Zelaznik, H. N., Smith, A., Franz, E. A., \& Ho, M. (1997). Differences in bimanual coordination associated with stuttering. Acta Psychologica, 96, $229-243$.

Received December 16, 1997

Revision received May 17, 1999 Accepted July 6, 1999 